\title{
Editorial (Heft 1, 2015)
}

\section{Ferdinand Eder $\cdot$ Herbert Altrichter $\cdot$ Angelika Paseka}

Die Beiträge des vorliegenden Heftes der ZBF legen einen Schwerpunkt auf den Umgang der Schule mit Ungleichheit bzw. Verschiedenheit. Angesprochen werden - mit durchwegs neuem Fokus - die schon klassischen Ungleichheitsmerkmale wie soziale Herkunft, Geschlecht oder Migrationshintergrund; es kommt jedoch auch zu einer Erweiterung der Perspektive, insofern auch die von den Schülerinnen und Schülern wahrgenommene Verschiedenheit von Lehrpersonen, speziell im Hinblick auf Migrationshintergrund, zum Thema wird.

In den Schulen der deutschsprachigen Bildungssysteme besteht ein Missverhältnis zwischen der Zahl von Schülerinnen und Schülern mit Migrationsherkunft auf der einen Seite und von Lehrkräften mit Migrationsherkunft auf der anderen Seite. Von einer Steigerung des Anteils solcher Lehrpersonen wird sowohl eine Verbesserung der schulischen Leistungen als auch der gesellschaftlichen Integration von Schülerinnen und Schülern mit Migrationsherkunft erwartet. Allerdings ist bislang weithin unerforscht geblieben, wie solche Lehrkräfte von den Lernenden selbst wahrgenommen werden. Carolin Rotter versucht mit ihrem Beitrag „Lehrkräfte mit Migrationshintergrund aus der Perspektive von Schülerinnen und Schülern“ diese Forschungslücke zu bearbeiten und stellt die Ergebnisse von qualitativen Gruppendiskussionen vor. In diesen zeigt sich eine durchaus differenzierte Wahrnehmung der Lehrenden durch die Jugendlichen, für die die Autorin eine Typenbildung vornimmt und zur Diskussion stellt.

\section{F. Eder $(\square)$}

Salzburg, Österreich

E-Mail: ferdinand.eder@sbg.ac.at

H. Altrichter

Linz, Österreich

A. Paseka

Hamburg, Deutschland 
Neda Forghani-Arani, Corinna Geppert und Tamara Katschnig greifen in ihrem Beitrag „Wenn der Pygmalioneffekt nicht greift..." auf Forschungsergebnisse aus Österreich zurück, die an den dortigen Neuen Mittelschulen erhoben wurden. Im Zentrum steht dabei die Frage, wie mit dem Heterogenitätsmerkmal Migrationshintergrund an diesen Schulen umgegangen wird. Der Umgang mit migrationsbedingter Heterogenität irritiert die Lehrkräfte und löst subtile Assoziationen aus, die Erklärungen für das migrationsbedingte Leistungsgefälle liefern können. Gleichzeitig treten erwartete Zusammenhänge nicht ein und die Autorinnen resümieren: „Der Pygmalioneffekt lässt uns im Stich!“ Im Lichte der Critical-Race-Theory lässt sich dieses Ergebnis dahingehend interpretieren, dass Differenz- und Defizitzuschreibungen wirksam werden, die jedoch nicht ein- sondern mehrdimensional zu denken sind.

Im Beitrag von Belinda Aeschliman, Walter Herzog und Elena Makarova über „Frauen in MINT-Berufen: Retrospektive Wahrnehmung des mathematisch-naturwissenschaftlichen Unterrichts auf der Sekundarstufe I“ geht es um das Differenzkriterium Geschlecht. Im Zentrum stehen Frauen, die sich für einen MINT-Beruf entschieden haben, und wie diese retrospektiv den selbst erfahrenen mathematischnaturwissenschaftlichen Unterricht in der Sekundarstufe I einschätzen. Dabei kristallisierten sich vier Dimensionen heraus, die auf zentrale Merkmale von gutem Unterricht verweisen: individuelle Förderung durch Unterstützung und Ermutigung, Orientierung an der Alltagserfahrung der Schülerinnen und Schüler, Vermittlungskompetenz der Lehrenden und eine Orientierung hin auf mögliche Zukunftsperspektiven.

Imke Groeneveld und Michael Knigge nähern sich im Beitrag „Moderation primärer sozialer Disparitäten im Leseverständnis in Abhängigkeit vom wahrgenommenen Verhalten der Lehrkraft und der Klassenzusammensetzung“ der Frage nach dem Zusammenhang zwischen sozialer Herkunft und schulischer Kompetenzentwicklung aus einer originellen Perspektive, indem sie anhand von Daten der längsschnittlichen Berliner Grundschulstudie ELEMENT nach Gründen suchen, warum einige Schülerinnen und Schüler „,erwartungswidrig“ höhere Kompetenzen zeigen, als ihre soziale Herkunft vermuten ließe. Die Ergebnisse legen nahe, dass Schülerinnen und Schüler aus Elternhäusern mit niedrigem sozioökonomischen Status besonders von der Zugehörigkeit zu einer Klasse mit höherem durchschnittlichen Sozialstatus profitieren. Die erhobenen Unterrichtsmerkmale (wie störungsarmer Unterricht mit individueller Förderung) weisen dagegen keinen moderierenden Effekt auf den Zusammenhang zwischen sozialer Herkunft und Kompetenzentwicklung im Lesen auf.

Susanne Schwab und Susanne Seifert gehen in ihrem Beitrag über „Einstellungen von Lehramtsstudierenden und Pädagogikstudierenden zur schulischen Integration“ der Frage nach, wie sich Studierende verschiedener Lehrämter untereinander sowie von Studierenden der Pädagogik in ihrer Einstellung zu einer inklusiven Schule unterscheiden. Die Ergebnisse sind durchaus überraschend, insofern kaum Unterschiede zwischen den verschiedenen Lehramtsstudienrichtungen bestehen, und stützen die Hoffnung, dass eine inklusive Schule zumindest von den nachwachsenden Lehrpersonen quer über die Schulformen hinweg unterstützt würde.

Netzwerke finden gegenwärtig als innovativer Steuerungsmodus für schulische Entwicklungen in der Forschung Beachtung. Doch wie werden diese selbst gesteuert? Robin Junker und Niels Berkemeyer versuchen diese Frage in ihrem Beitrag „Netzwerkbeziehungen und Steuerungsstrukturen: Eine Analyse interpersonaler 
Beziehungsmuster im schulischen Netzwerk Hochbegabtenförderung NRW“ die Steuerungsmuster von zehn Netzwerkkoordinatoren sowie die Beziehungsmuster zwischen 74 Netzwerk-Teilnehmern im Rahmen von gemeinsamen Tagungen in fünf nordrhein-westfälischen Bezirksregierungen zu analysieren. Dabei überwiegt bei den Transaktionen zwischen den unterschiedlichen Ebenen der Steuerungsmodus „Beeinflussung“ gegenüber der Strategie „Verhandlung“, die entsprechend den Annahmen der Netzwerk-Konzeption erwartet worden wäre.

Alle Beiträge beziehen sich damit auf Herausforderungen, die in den aktuellen gesellschaftlichen Trends ihren Ursprung haben, und eine permanente Weiterentwicklung der Schule erfordern.

Das Heft enthält weiters eine Rezension von Franz Hofmann über den von Christina Schwer und Claudia Solzbacher herausgegebenen Sammelband Professionelle pädagogische Haltung. Historische, theoretische und empirische Zugänge zu einem viel strapazierten Begriff, sowie von Josef Thonhauser über den vierten Band der von Wolfgang Brezinka verfassten Pädagogik in Österreich. Die Geschichte des Faches an den Universitäten vom 18. bis zum 21. Jahrhundert. Band 4: Pädagogik an der Wirtschaftsuniversität Wien und der Universität Klagenfurt.

Und auch diesmal wird das Heft durch Nachrichten aus der ÖFEB abgerundet. 